\title{
Intranasal nanoemulsion vaccine confers long-lasting immunomodulation and sustained unresponsiveness in a murine model of milk allergy
}

\author{
Jessica J. O'Konek (1) | Jeffrey J. Landers | Katarzyna W. Janczak | Hayley K. Lindsey | \\ Anna M. Mondrusov | Tiffanie D. Totten | James R. Baker Jr. (C)
}

Mary H. Weiser Food Allergy

Center, University of Michigan, Ann Arbor, MI, USA

\section{Correspondence}

Jessica J. O'Konek, Mary H. Weiser Food Allergy Center, University of Michigan, 4341 Biomedical Sciences Research Building, 109 Zina Pitcher Place, Ann Arbor, MI, USA.

Email: jjoz@umich.edu

\section{Funding information}

Food Allergy Research and Education New Investigator Award; Michigan Food Allergy Research Accelerator (M-FARA); Robert and Caren Vondell

\begin{abstract}
Background: Immunotherapy for food allergy requires prolonged treatment protocols and, in most cases, does not lead to durable modulation of the allergic immune response. We have demonstrated an intranasal (IN) nanoemulsion adjuvant that redirects allergen-specific Th2 responses toward Th1 and Th17 immunity, and protects from allergen challenge after only 2-4 monthly administrations. Here, we investigate the ability of this technology to provide long-term modulation of allergy in a murine model of cow's milk allergy.

Methods: Six weeks after sensitization to bovine casein, mice received four, monthly IN immunizations with nanoemulsion formulated with casein. Protection from casein challenge was assessed at 4 and 16 weeks after the final vaccine administration.

Results: The NE vaccine significantly blunted the physiological responses to allergen challenge, and this effect persisted for at least 16 weeks. The protection from challenge was associated with the suppression of casein-specific Th2 immunity and induced Th1 and Th17 cytokines as well as induction of IL-10. Of interest, while immunized animals showed significantly decreased Th2 cytokine responses, cow's milkspecific IgE remained elevated in the serum at levels associated with reactivity in control sensitized animals. Protection was associated with suppressed mast cell activation and markedly reduced mast cell infiltration into the small intestine.

Conclusion: The sustained unresponsiveness of at least 16 weeks after vaccination suggests that the nanoemulsion vaccine alters the allergic phenotype in a persistent manner different from traditional desensitization, and this leads to long-term suppressive effects on allergic disease without eliminating serum IgE.
\end{abstract}

\section{KEYWORDS}

allergy treatment, food allergy, immunotherapy and tolerance induction, immunotherapy vaccines and mechanisms, vaccines 


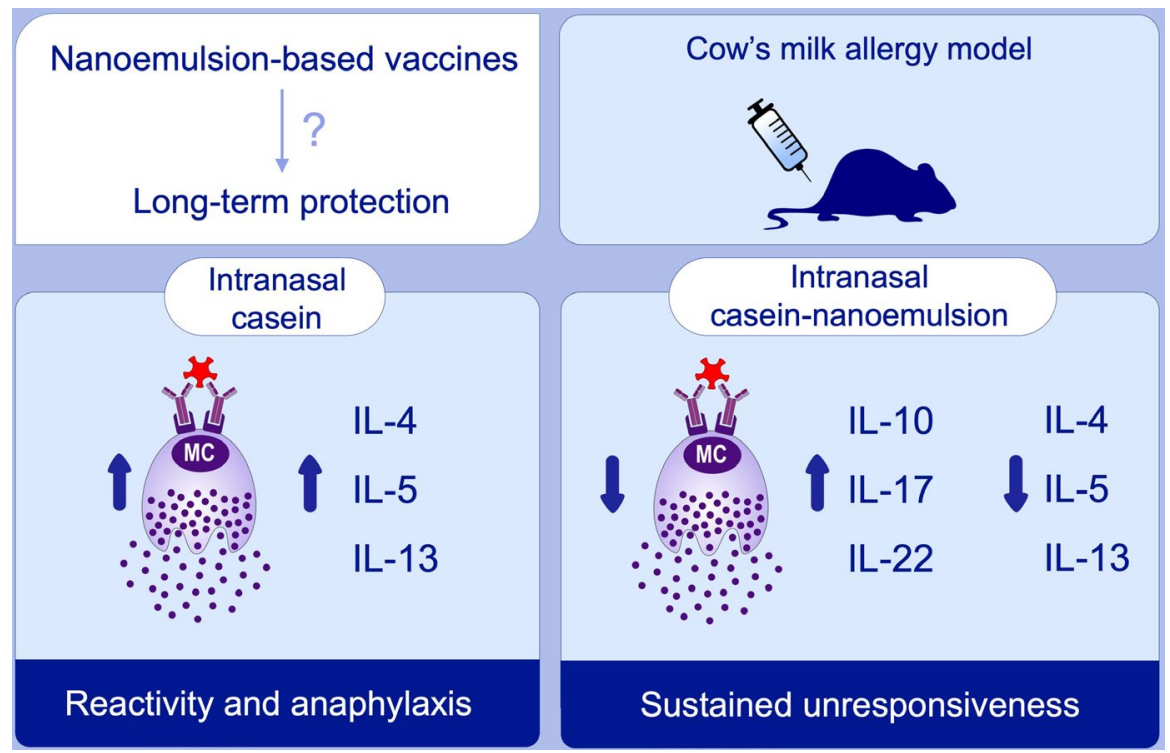

\section{GRAPHICAL ABSTRACT}

This study evaluates the ability of an intranasal nanoemulsion-based vaccine to induce long-term modulation of allergic reactions in a mouse model of cow's milk allergy. Intranasal immunization with nanoemulsion adjuvant suppresses Th2 responses and anaphylaxis. The sustained unresponsiveness of at least 16 weeks after vaccination suggests that the nanoemulsion vaccine alters the allergic phenotype.

\section{1 | INTRODUCTION}

Food allergies have become common in industrialized nations and affect an estimated $5 \%$ of adults and $8 \%$ of children in the United States. ${ }^{1,2}$ Milk is one of the eight major allergens responsible for the majority of serious food allergy reactions in the United States, and allergy to cow's milk is the most common food allergy in infants and young children. ${ }^{3}$ While cow's milk allergy is often outgrown, high milk-specific IgE in infants is associated with persistent milk allergy as well as increased incidence of atopic dermatitis, asthma, rhinoconjunctivitis, and other food allergies. ${ }^{4,5}$

Strict avoidance of an offending food is the primary clinical approach to prevent allergic reactions and anaphylaxis, often coupled with the use of epinephrine for accidental exposure. Recently, however, progress has been made toward the development of immunotherapy for food allergy. This includes oral (OIT), sublingual (SLIT), and epicutaneous (EPIT) immunotherapies (reviewed $\mathrm{in}^{6,7}$ ), and single-allergen OIT for peanut allergy has been submitted to the FDA for approval. ${ }^{8-10}$ OIT has also been studied for milk allergy in numerous clinical trials. ${ }^{11}$ However, most evidence suggests that OIT is not effective to induce tolerance or durable sustained unresponsiveness (SU) to food allergens. SU, as defined by the ability to pass a double-blind, placebo-controlled food challenge (DBPCFC) 2-8 weeks after cessation of the immunotherapy, has been reported to occur in $25 \%-58 \%$ of trial participants, ${ }^{12-14}$ and this included patients that may have naturally outgrown their allergy. Thus, while these immunotherapies have demonstrated efficacy, this lack of sustained protection suggests that OIT in most cases does not fundamentally change the allergen-specific allergic response.
An optimal goal for treating food allergies would be to generate immune tolerance in allergic individuals and suppress the underlying Th2-polarized cellular immune responses, including IL-4 and IL-13, and allergen-specific IgE antibodies. ${ }^{1,15-17}$ Recent work has demonstrated that the induction of tolerance in allergies is associated with both the suppression of mast cell and basophil reactivity and changes in allergenspecific cells, including the upregulation of regulatory markers and increases in the number and/or function of regulatory T cells (Tregs). Thus, the goal of approaches for long-term SU or tolerance to prevent allergic reactions could include both the durable suppression of allergen-specific Th2 immunity and reductions in number and reactivity of effector cells.

Our group has developed an intranasal nanoemulsion (NE) adjuvant that modulates preexisting allergen-specific Th2 immune responses toward a Th1 and Th17 phenotype. ${ }^{18}$ We previously reported that in murine models of peanut allergy, this modulation resulted in protection when mice were challenged with peanut 2-3 weeks after final immunization. ${ }^{19}$ As adjuvants are effective in inducing long-term changes in the immune response, we hypothesized that these NE-based vaccines might stably modulate the allergen-specific immune response resulting in durable SU. In this study, we test the ability of an intranasal NE-based vaccine to induce longterm modulation of established Th2 immunity and SU from allergic reactions in a mouse model of cow's milk allergy.

\section{2 | MATERIALS AND METHODS}

\section{1 | Antigen and adjuvants}

Nanoemulsion adjuvant (NE) was produced by a high-speed emulsification of ultra-pure soybean oil with cetylpyridinium chloride, 
(A)

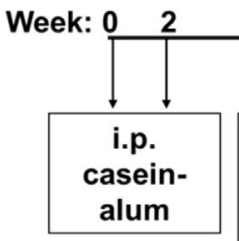

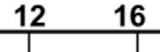

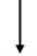

i.n.

- casein-NE

- casein-PBS

- PBS
(B)

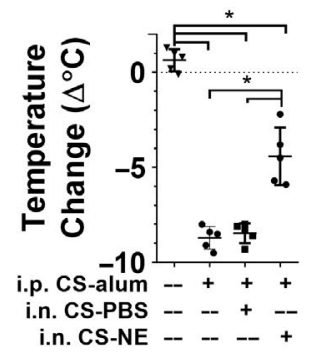

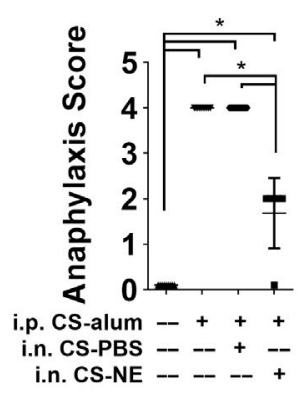

(C)

Week 36 (16-wk SU)

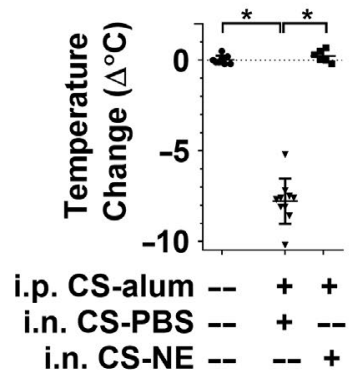

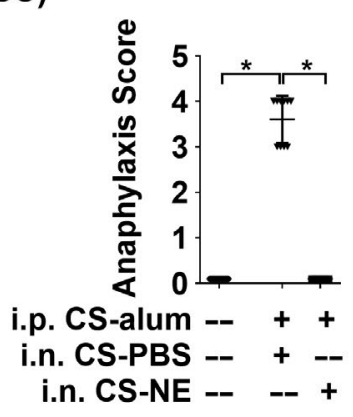

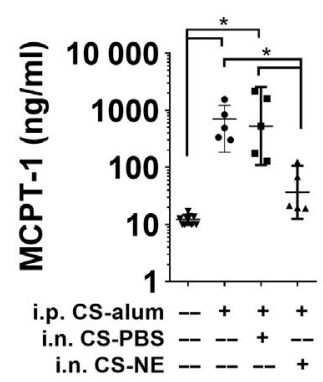

FIGURE 1 Protection conferred by $\mathrm{NE}$ immunization is sustained for at least 16 wk. (A) Mice were sensitized with casein-alum (CS-alum) and immunized i.n. with 4 administrations of casein-NE (CS-NE) or casein-PBS (CS-PBS). Mice were challenged intraperitoneally with casein at (B) week 24, $4 \mathrm{wk}$ after the final i.n. immunization, or (C) week 36, 16 wk after the final i.n. immunization. In order to assess sustained unresponsiveness $\mathrm{SU})$, the degree of allergic reactions was measured by change in core body temperature, clinical anaphylaxis score, and serum MCPT-1 levels as determined by ELISA. Statistically significant differences $(P<.05)$ are indicated by *

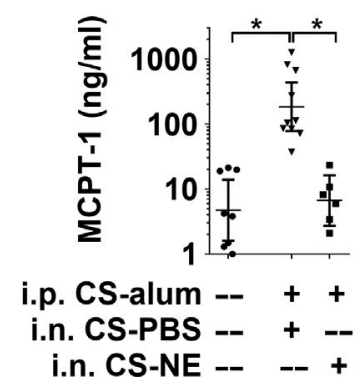

Tween 80 and ethanol in water, with resultant NE droplets with average 350-400 nm diameter. ${ }^{20,21}$ Aluminum hydroxide (alum, alhydrogel) was purchased from InvivoGen. Casein purified from bovine milk, containing the four main types of casein found in cow's milk: $\alpha$ s1 Casein, $\alpha$-s2 Casein, $\beta$-Casein, and $\kappa$-Casein, was purchased from Sigma and was solubilized in phosphate buffer saline (PBS) and sterilized by sequential filtration through $0.4-$ and $0.2-\mu \mathrm{m}$ syringe filters. Endotoxin content of all vaccine components was determined by a limulus amebocyte lysate ( $L A L$ ) assay (Pierce). There was no detectable endotoxin in PBS, NE, or alum (Figure S1). The stock solution of casein was approximately 5 endotoxin units (EU) per $\mathrm{mL}$; however after dilution into the final vaccine formulation, the total amount of endotoxin given intranasally to each mouse is $0.004 \mathrm{EU}$ per dose. As $0.004 \mathrm{EU}$ is equivalent to approximately $0.4 \mathrm{pg}$ of LPS, this amount of endotoxin is not anticipated to have major effects.

\subsection{Mice and immunizations}

Specific pathogen-free BALB/c mice (females 3 weeks old) were purchased from Jackson Laboratory. Mice were 4 weeks of age at the onset of the experiment. The experimental design is shown in Figure 1. Allergic sensitization was induced with intraperitoneal immunizations (i.p.) of $125 \mu \mathrm{g}$ bovine casein (Sigma) adsorbed on $1 \mathrm{mg}$ alum at weeks 0 and 2. Intranasal (i.n.) immunizations were administered as $12 \mu \mathrm{L}$ ( $6 \mu \mathrm{L} /$ nare) of a formulation containing $20 \mu \mathrm{g}$ of casein mixed with $20 \%$ NE. Casein mixed with PBS alone served as a control. Systemic anaphylaxis was induced by i.p. injection of $100 \mu \mathrm{g}$ casein, and reactions were assessed as described below. For oral challenge experiments, mice were fasted for 5-6 hours to ensure gastric emptying and then were challenged with $0.2 \mathrm{~mL}$ of $4 \%$ fat cow's milk by oral gavage. Mice were challenged orally every other day for a total of 7 gavages. ${ }^{22}$ All animal procedures were performed according to the University of Michigan Institutional Animal Care and Use Committee and the National Institutes of Health guide for the care and use of laboratory animals.

\section{3 | Assessment of hypersensitivity reactions}

Anaphylactic symptoms were evaluated for 1 hour following challenge using the following scoring system (modified from ${ }^{23,24}$ ): 0 , no symptoms; 1 , prolonged rubbing and scratching around the nose, eyes, or head; 2 , puffiness around the eyes or mouth, diarrhea, piloerection, and/or decreased activity with increased respiratory rate; 3 , labored respiration, wheezing, stridor, and/or cyanosis around the mouth and tail; 4, tremor, convulsion, no activity after prodding and/or moribund; and 5, death. Rectal temperature was monitored for 60 minutes following challenge. Mice were bled 60 minutes 
following challenge, and serum mouse mast cell protease-1 (MCPT-1) was determined by ELISA (eBioscience).

\subsection{Measurement of serum antibodies}

Sera were obtained by saphenous vein bleeding or by cardiac puncture posteuthanasia. Serum was separated from whole blood by centrifugation at $1500 \times \mathrm{g}$ for 5 minutes after allowing coagulation for 30-60 minutes at room temperature. Serum samples were stored at $-20^{\circ} \mathrm{C}$ until analyzed. Casein- and cow's milk-specific IgE antibodies were determined by ELISA as described previously. ${ }^{19}$ Briefly, serially diluted serum samples were incubated on microtiter plates coated with $20 \mu \mathrm{g} / \mathrm{mL}$ bovine casein or cow's milk. IgE, IgG1, and IgG2a were detected with alkaline phosphatase conjugated anti-mouse IgE (Rockland) and IgG1 and IgG2a (Jackson ImmunoResearch) antibodies and Sigma Fast ${ }^{\text {TM }} \mathrm{p}$ nitrophenyl phosphate substrate (Sigma) and quantified by measuring the optical density (OD) at $405 \mathrm{~nm}$. The antibody concentrations are presented as endpoint titers defined as the reciprocal of the highest serum dilution producing an OD above background of naïve sera. The cutoff value is determined as the OD (mean +2 standard deviations) of the corresponding dilution of naive sera. ${ }^{25,26}$

\section{5 | Analysis of cytokine production}

The cellular recall response was evaluated in lymphocytes isolated from mesenteric lymph nodes. Single cell lymphocyte suspensions were cultured ex vivo \pm casein $(25 \mu \mathrm{g} / \mathrm{mL})$ at $37^{\circ} \mathrm{C}$. After 72 hours, cytokine secretion was measured in cell culture supernatants using Luminex multiplex detection system (Millipore).

\subsection{Acute allergic skin response}

The acute allergic skin response (ear swelling at 1 hour) was determined in anesthetized mice after intradermal (i.d.) injection of $10 \mu \mathrm{g}$ casein in the ear pinnae. Ear thickness was measured in duplicate using a digital micrometer. The allergen-specific change in ear swelling was compared with the nonspecific ear swelling due to allergen injection in the PBS (sham)-sensitized group.

\section{7 | Mast cell quantification}

Eighteen hours after challenge, the duodenum and jejunum were fixed in $10 \%$ formalin, embedded in paraffin, and cut into $5-\mu \mathrm{m}$ thick sections. Tissue sections were stained for chloroacetate esterase (CAE) activity as previously described. ${ }^{22,27}$ Quantification of mast cells was performed by counting the number of CAE-positive cells from at least 25 fields of view at $40 \times$ magnification, and the data are reported as number of cells per field.

\subsection{Serum transfer experiments}

Mice were sensitized i.p. with casein and alum at weeks 0 and 2 and immunized i.n. with casein-NE or casein-PBS at weeks 6,12
18, and 20 as described above and in Figure 1. At week 24, mice were killed and serum was harvested. Serum from mice receiving the same treatments was pooled and transferred into naïve recipient mice ( $200 \mu \mathrm{L} /$ mouse) by injection into the tail vein. Twenty-four hours after serum transfer, recipient mice were challenged orally with cow's milk to assess reactivity.

\section{9 | Statistics}

Statistical comparisons were assessed by the Mann-Whitney test using GraphPad Prism version 7 (GraphPad Software). The $P$ value $<.05$ was considered as significant. Results presented here are the representatives of at least two independent experiments.

\section{3 | RESULTS}

\section{1 | Intranasal immunization with NE adjuvant suppresses allergic reactions and anaphylaxis}

$B A L B / c$ mice were sensitized to the cow's milk protein casein at weeks 0 and 2 by i.p. injections of bovine casein adsorbed on alum. ${ }^{19}$ Six weeks after sensitization, one group of mice received four, monthly i.n. immunizations with casein formulated in NE while the others received i.n. casein or PBS as controls. The mice were subsequently challenged intraperitoneally with casein to assess protection. At week 24, 4 weeks after the last i.n. immunization, sensitized control mice had profound reactions to challenge, indicated by core body temperature loss of $>5^{\circ} \mathrm{C}$ and severe symptoms of anaphylactic shock including pruritus, puffiness around the eyes and mouth, labored respiration or wheezing and lack of activity when prodded (Figure 1B). As compared to controls, the NE vaccine markedly suppressed physiological responses to allergen challenge. There was significant improvement in body temperature loss $(P=.003)$ as well as anaphylaxis symptom score $(P=.002)$, and mice that received the NE vaccine exhibited only mild symptoms of allergic reaction, as none experienced respiratory problems or shock. In order to quantify mast cell degranulation, MCPT-1 was measured in serum following challenge. Consistent with the clinical symptoms of allergic reaction, immunized mice had a significant reduction in MCPT-1, with average levels of $74 \mathrm{ng} / \mathrm{mL}$ compared with 528 and $840 \mathrm{ng} / \mathrm{mL}$ in control groups that received i.n. casein or PBS $(P=.0079)$. No significant differences in reactivity were observed in sensitized mice that received i.n. casein in PBS compared to sensitized mice that were not treated intranasally with allergen, demonstrating that the four i.n. exposures to $20 \mu \mathrm{g}$ casein did not significantly modulate reactivity. Similarly, i.n. administration of NE without allergen had no effect on the suppression of allergic reactivity (Figure S2).

In order to investigate durability of protection, mice were challenged 16 weeks after the final i.n. immunization. The increased time between sensitization and challenge did not influence the severity of allergic reactions for control mice (Figure 1C). In contrast, immunized mice were protected from challenge as body temperature loss, anaphylaxis symptom score, and MCPT-1 were significantly 
(A) Week 24
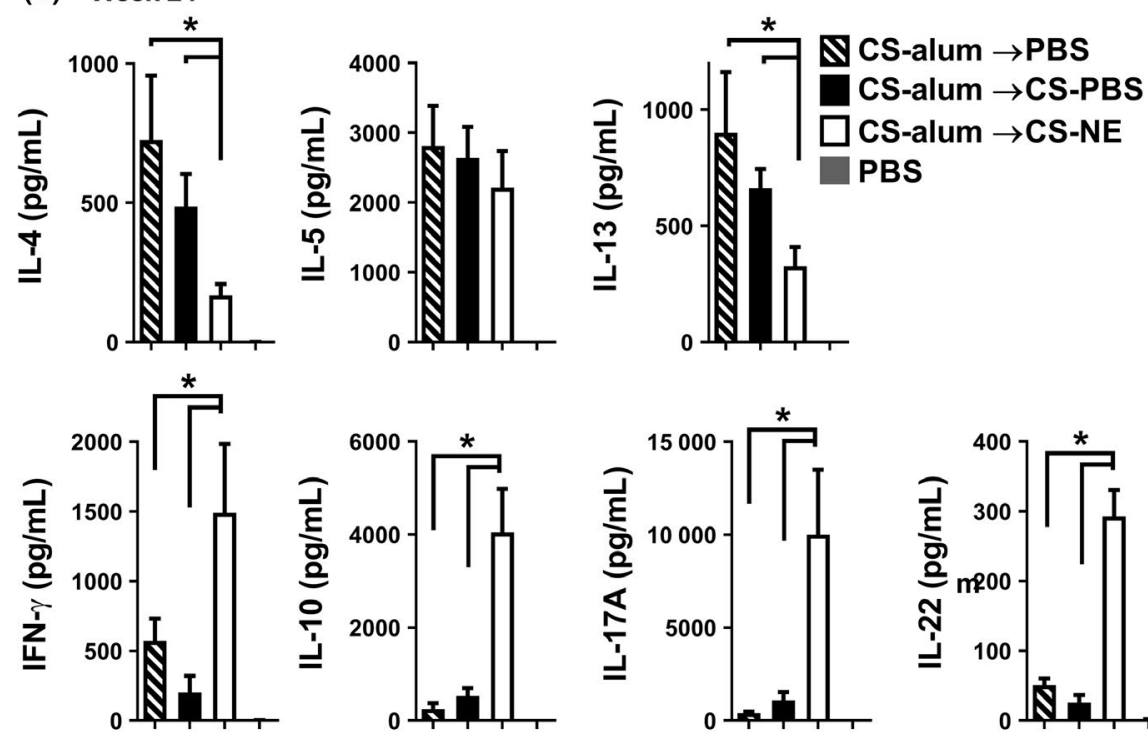

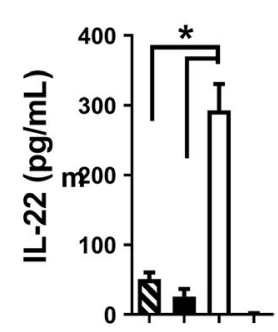

FIGURE 2 Nanoemulsion

immunization suppresses Th2 immunity and induces Th1/Th17 in mice sensitized to cow's milk protein. Cellular recall immune responses to bovine casein were measured in $\mathrm{mLN}$ lymphocytes harvested from mice at weeks (A) 24 and (B) 36. Cytokine secretion in culture supernatant was determined by a Luminex multiplex assay. Cytokine production has been normalized to control unstimulated lymphocyte cultures for each sample. Values are calculated as [stimulated] - [unstimulated] $=$ Total $(\mathrm{pg} /$ $\mathrm{mL}$ ) for each cytokine. Data are expressed as mean \pm standard deviation. Statistically significant differences $(P<.05)$ are indicated by *

(B) Week 36
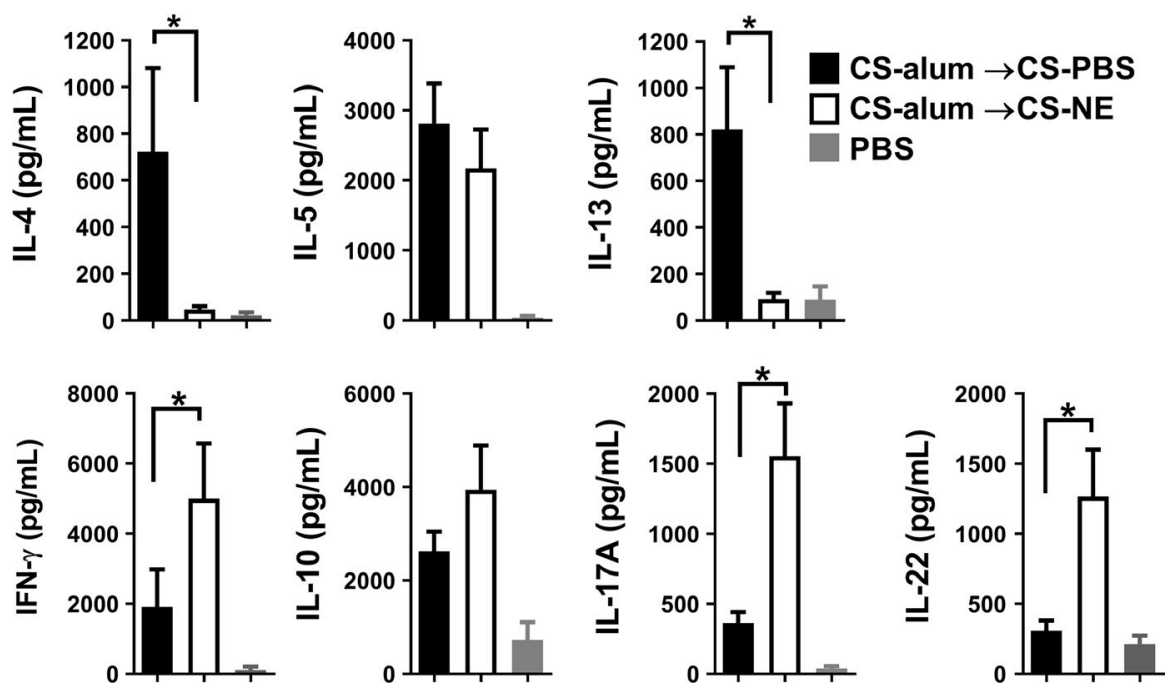

improved ( $P=.0001, .0002$, and .002 , respectively). The protection from challenge appeared complete, as there were no differences in reactions in NE-immunized mice compared with nonsensitized mice. This indicated that therapeutic immunization with NE results in complete protection 16 weeks after the final intranasal administration, with the protection appearing more pronounced than at earlier time points.

\section{2 | Intranasal immunization with NE adjuvant suppresses allergy-associated Th2 responses}

We have previously reported that allergen-NE immunization modulates allergen-specific Th2-polarized immunity while inducing IL-10, Th1, and Th17 immune responses. ${ }^{18,19}$ To confirm this in the milk allergy model, mesenteric lymph node cells were stimulated ex vivo with casein to characterize the recall response to allergen after the 4-week SU challenge at week 24 (Figure 2A). Upon stimulation

with casein, cells from casein-alum-sensitized mice produced predominantly Th2-type cytokines (IL-4, IL-5, IL-13) with lower levels of IFN- $\gamma$, IL-10, IL-17, and IL-22 (Figure 2A). Lymphocytes from mice that received subsequent casein-NE immunizations produced significantly more Th1 cytokines and significantly less Th2 cytokines. Casein-NE significantly increased IL-10 ( $P=.01)$ and the Th17 cytokines IL-17 and IL-22 ( $P=.0079)$ and decreased IL-4 and IL-13 $(P=.016$ and .032 , respectively). Consistent with reactivity data described above, i.n. administration of casein did not have any significant effects on cytokine production, compared with sensitized mice that were not treated i.n. with casein. Lymphocytes from PBS control mice (nonsensitized but challenged) did not produce measurable cytokine upon restimulation with casein.

Cytokine production was also characterized after the 16-week SU challenge (week 36) to determine whether modulation of the allergen-specific cytokine response persisted for 16 weeks after the final i.n. immunization. Consistent with the strong protection from 
(A)
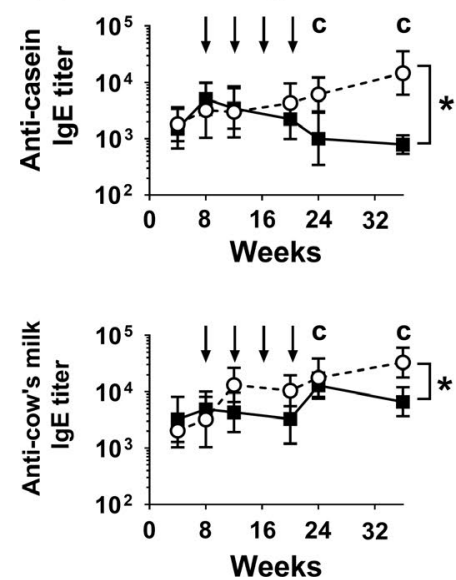

(B)
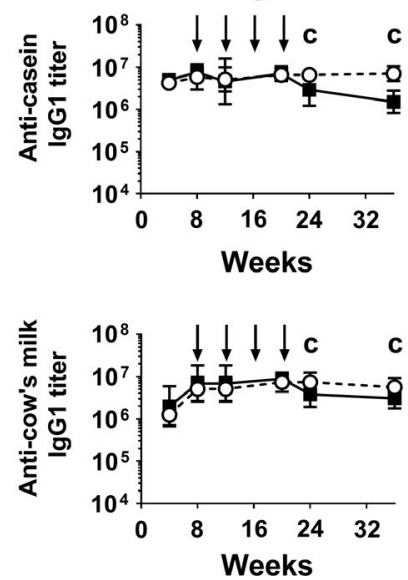

(C) IgG2a
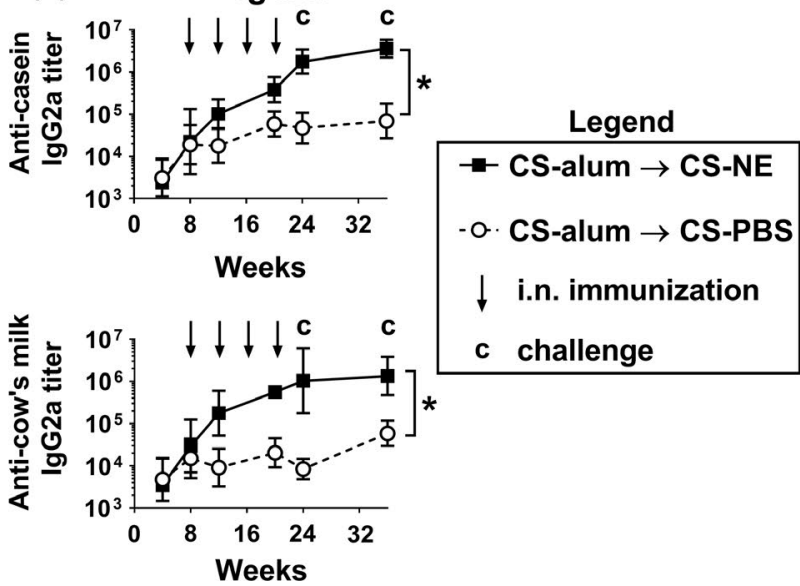

FIGURE 3 Nanoemulsion immunization modulates allergen-specific IgE and IgG in serum. Serum casein- and cow's milk-specific (A) $\operatorname{lgE}$, (B) IgG1, and (C) IgG2a were measured by ELISA throughout the course of the therapeutic protocol. Statistically significant differences $(P<.05)$ are indicated by *

challenge at this time point, IL-4 and IL-13 production remained significantly suppressed ( $P=.001$ and .007) (Figure 2B). Interestingly, from weeks 24 to 30 , there was an approximately 3.5 -fold reduction in IL-4 and IL-13 in the casein-NE-immunized animals, while the production of these cytokines was increased in the nonimmunized mice possibly as a result of reactivity upon challenge.

Nanoemulsion immunization-induced enhancement of IFN- $\gamma$, IL-17, and IL-22 was maintained at week 36 ( $P=.042, .01$ and .0007). However, at this later time point, there was no significant difference in IL-10 production between mice that received i.n. instillations of casein-NE or casein-PBS. While IL-10 production remained constant at approximately $4000 \mathrm{ng} / \mathrm{mL}$ in mice that received casein-NE, there was a fivefold increase in IL-10 production in mice receiving casein-PBS. For all cytokines tested, there was largely no detectable antigen-specific cytokine production in the nonsensitized control group at week 24, and all cytokines increased at week 36 to low, but detectable, levels. This suggests minimal effect from the prior casein challenge on casein-specific cytokine production at week 36 in nonsensitized animals. Overall, these data demonstrate that the modulation of Th2 cytokine production not only remains modulated for at least 16 weeks after the final immunization but also becomes stronger in the months after immunization.

\subsection{NE reduces mast cell infiltration into tissue and protects despite the presence of allergen-specific IgE in the serum}

Allergen-specific antibodies were quantified from the serum throughout the course of the study (Figure 3). Sensitization and immunization with casein induced similar levels of antibodies specific for casein and cow's milk, demonstrating that these antibodies bind to casein epitopes that are present in cow's milk. Immunization with casein-NE largely prevented further increases in allergen-specific IgE that occurred in nonimmunized mice. Surprisingly, at week 24 despite being protected from challenge, serum casein- and cow's milk-specific IgE titers were not significantly different in casein-NE- and casein-PBS-immunized mice (Figure 3A). At week 36, allergen-specific IgE was significantly decreased in the serum of NEimmunized mice ( $P=.007)$; however, IgE antibody titers remained significantly elevated at titers where nonimmunized mice would be expected to react to challenge (Figure $3 \mathrm{~A}$ ). While allergen-specific IgG1 titers were not significantly affected by the casein-NE treatment (Figure 3B), IgG2a titers were increased more than 20 -fold in the serum of mice that received casein-NE $(P<.0001)$, demonstrating a shift in the profile of cow's milk-specific antibodies (Figure $3 \mathrm{C}$ ).

To confirm that the NE vaccine could protect from IgE-mediated allergic reactions, the acute allergic skin response to intradermal injection of casein was measured. NE treatment reduced the acute allergic skin responses compared to the casein-sensitized control mice (Figure 4B). As this further suggested that the NE-immunized mice were protected from IgE-mediated allergic reactions, we tested the ability of NE to protect from reactivity in an additional experimental food allergy model in which intestinal mast cells drive the clinical phenotype. ${ }^{22,28,29}$ As illustrated in Figure 4A, 2 weeks after the final i.n. immunization, mice were challenged orally with cow's milk every other day for 2 weeks. NE-immunized mice were significantly protected from allergic reaction to oral cow's milk challenge, as indicated by a >10-fold reduction in MCPT-1 $(P=.0002)$ (Figure $4 C$ ). Mast cells were quantified in the small intestine to determine whether NE immunization protected through blocking the accumulation of mast cells in the tissues. There was an almost 10fold increase in small intestine mast cells in sensitized mice; however, mast cell infiltration was significantly lower in NE-immunized mice $(P<.0001)$ and was identical to mast cell numbers observed in nonsensitized mice (Figure 4D and Figure S3). These data suggest that while allergen-specific IgE circulates in the blood, the NE-immunized mice are protected from challenge due to the lack of mast cell accumulation in the tissue.

In order to further assess the role of the humoral immune response in the protection conferred by NE immunization, a passive 


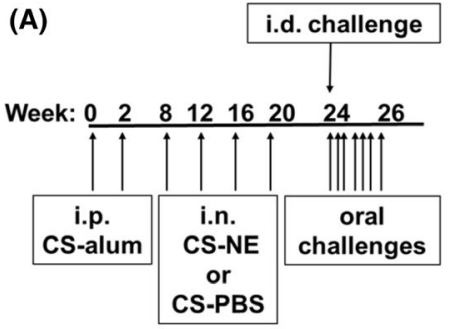

(C)

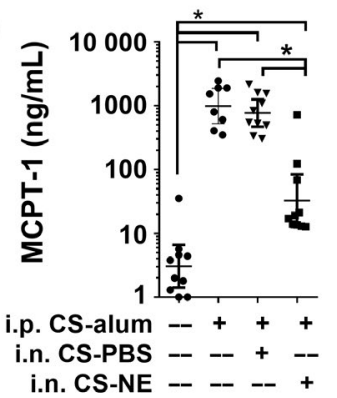

(B)

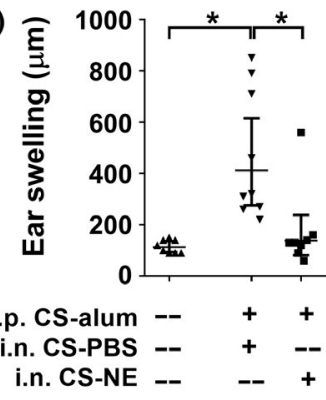

(D)

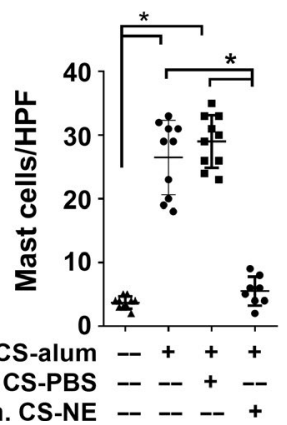

FIGURE 4 Nanoemulsion immunization protects from mast cell-mediated allergic reactions. (A) Mice were sensitized with casein-alum (CS-alum) and immunized i.n. with 4 administrations of casein-NE (CS-NE) or casein-PBS (CS-PBS). At week 24, mice were challenged either (B) intradermally (i.d) with casein or (C) orally with cow's milk for a total of 7 challenges over 2 wk. (B) The effect of $\mathrm{NE}$ immunization on the acute allergic skin response was measured before and $1 \mathrm{~h}$ after the i.d. challenge with casein. The effect of NE immunization on allergic reaction to oral challenged was measured by $(C)$ serum MCPT-1 levels and (D) the number of mast cells in the jejunum after the 7th oral cow's milk challenge. Histology images were obtained at $40 x$ magnification and cells counted per field of vision. Statistically significant differences $(P<.05)$ are indicated by *

sensitization experiment was performed. Serum was harvested from mice following sensitization and immunization and transferred into naïve recipient mice. Recipient mice were challenged to determine whether the serum from NE-immunized mice would confer reactivity. Mice that received serum from sensitized mice that were immunized with casein-PBS reacted to challenge, as demonstrated by a decrease in core body temperature and increase in MCPT-1 (Figure 5). Mice that received serum from casein-NE-immunized mice had significantly reduced reactions. These data demonstrate that while serum in these mice contains allergen-specific lgE, it does not confer sensitively to the allergen.

\section{4 | DISCUSSION}

To date, no immune approach to address food allergies has demonstrated long-term protection from reactivity, identified as SU, which is an unmet need for the millions of patients with food allergies. We have investigated the use of adjuvants to induce a durable modulation of allergen-specific immune responses that is effective with few immunizations. Our previous results demonstrated that NE-based

(A)

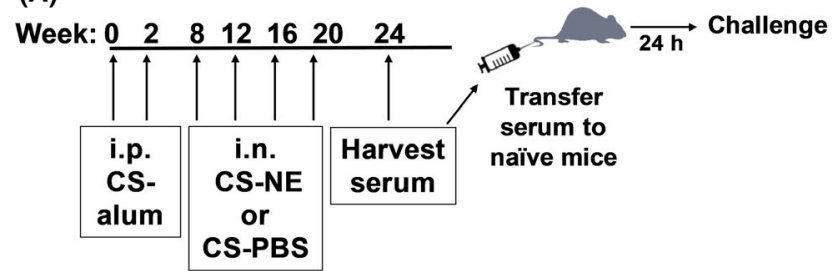

(B)

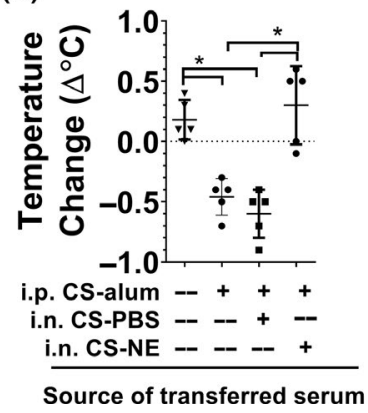

(C)

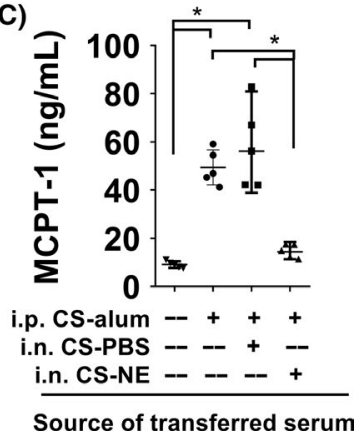

FIGURE 5 Serum from NE-immunized mice does not induce passive sensitization in recipient mice. (A) Serum from caseinsensitized mice that received i.n. PBS, casein-PBS or casein-NE was harvested at week 24 and transferred into naïve recipient mice. Twenty-four hours later, recipient mice were challenged orally with cow's milk and reactivity was measured by (B) change in body temperature and $(C)$ serum MCPT-1 levels. Statistically significant differences $(P<.05)$ are indicated by *

allergy vaccines could suppress peanut allergy in several mouse models a few weeks after administration of the last immunization. The present studies investigate whether the immunomodulation of the allergen-specific response by NE vaccine could induce durable immune alterations capable of conferring long-term SU and protection from allergen challenge. We demonstrated that NE-immunized mice were protected for 16 weeks following NE immunization and these mice were completely unresponsive to allergen challenge at that late time point. Protection from allergen challenge correlated with continued suppression of allergen-specific Th2 immune responses, suggesting that the changes in the allergic immune phenotype were also durable.

While allergen-specific IgE is commonly used to diagnose food allergies, many patients with allergen-specific IgE in the blood do not react upon consumption of that food. ${ }^{30,31}$ In this study, while cow's milk-specific IgE was significantly decreased in the serum of $\mathrm{NE}$-immunized mice at the end of the study, IgE titers remained significantly elevated to similar levels where sensitized control (non-NE vaccinated) mice reacted to challenge. Since these NE-immunized mice were protected from IgE-mediated allergic reactions, this suggests that similar to food allergic humans, serum allergen-specific IgE is not completely predictive of clinical reactivity. In addition, while serum casein-specific IgE was not completely suppressed in NE-immunized mice, there was complete suppression of mast cell accumulation in the intestine after oral challenge, consistent with reports that food allergic reactions in murine models of food allergy are dependent upon tissue mast cell infiltration. ${ }^{22,28}$ 
Serum transfer from NE-immunized mice was used to dissect the humoral and cellular immune mechanisms responsible for the protection induced by the NE vaccines. While transfer of serum from sensitized and nonimmunized mice passively sensitized mice, serum from sensitized and NE-immunized mice did not result in reactivity in recipient mice. The serum from NE-immunized mice contained allergen-specific IgE; however, the transfer of this IgE did not transfer reactivity. This suggests that the modulation of the allergen-specific antibody profile may play a key role in the protection from challenge observed following NE immunization. Modulation of the humoral immune response can lead to protection through multiple mechanisms. NE immunization may induce blocking antibody that can neutralize the allergen before it binds to IgE on effector cells. ${ }^{32,33}$ Mast cell degranulation can also be suppressed through the activation of the inhibitory Fc receptor FcyRIlb. ${ }^{33-35}$ Allergen-specific IgG2a is significantly increased in NE-immunized mice at both the 24- and 36-week challenges and may be involved in the inhibition of mast cell activation either through the activation of Fc $\gamma$ RIlb or by blocking allergen from binding to IgE. Together, these data suggest that while allergen-specific IgE circulates in the blood, NE-immunized mice may be protected from clinical reactions due to reduced mast cell recruitment, accumulation, and activation in the gut. These results also suggest that tissue mast cell infiltration would be a strong predictor of food allergy reactivity compared to allergen-specific serum IgE.

Other studies have demonstrated efficacy of immunotherapies for food allergies in mice but have typically challenged mice within 2 weeks of the last therapeutic intervention. ${ }^{36-45}$ As this is a timepoint where the effect of desensitization could not have worn off, we modified this approach by evaluating $\mathrm{SU}$ in excess of 3 months after discontinuation of therapy. While our studies were performed in mice, comparisons to studies of OIT performed in both mice and humans can provide insights into how this might translate into protection in humans. Studies of OIT in mice have demonstrated that protection is lost 2 weeks after stopping $\mathrm{OIT}^{46}$ In humans, however, OIT has led to SU intervals of 2-8 weeks in a fraction of patients. Despite this, most OIT human trials also have shown that as time posttreatment increases the percentage of patients who pass the DBPCFC decreases. ${ }^{47,48}$ Thus, it is significant that the NE vaccines induce protective immunity that persists in all mice for at least 16 weeks off treatment. It also suggests that the complete unresponsiveness at 16 weeks after the last immunization in mice could translate into a significant duration of protection in humans.

Desensitization and tolerance are two distinct outcomes of immunotherapy, and it is important to distinguish between the two. Desensitization is an increase in the threshold amount of food that can be ingested without reaction while on the therapy. Desensitization is mediated by changes in effector cells, such as mast cells, but does not involve modulation of the underlying allergen-specific immune mechanisms. Thus, desensitization is temporary and the individual remains allergic to the allergen, reacting to the antigen soon after the therapy is stopped. Conversely, true tolerance is more permanent protection from reaction after discontinuation of the immunotherapy. Tolerance involves modulation of effector cells as well as allergen-specific immune cells, typically through the induction of regulatory mechanisms. ${ }^{49,50}$ While the sustained unresponsiveness observed with OIT is likely mostly due to desensitization, the durable protective immunity and immunomodulation induced by the NE vaccines presented here are not consistent with desensitization and suggest an immune phenotype more consistent with tolerance.

We have previously reported that therapeutic immunization with NE induces Tregs. ${ }^{18,19}$ Increases in both Tregs and IL-10 suggest that the suppression of Th2 immunity and subsequent protection from allergen challenge is due to the induction of regulatory mechanisms. A similar induction of Tregs and IL-10, as well as IFN- $\gamma$, has been reported in grass pollen allergic individuals with decreased Th2 immune responses after allergen immunotherapy. ${ }^{51,52}$ The induction of IL-10 is not sufficient for the suppression of reactivity, as in this study casein-sensitized mice that received i.n. casein-PBS retain reactivity at week 36 despite having increased IL-10. This suggests that the induction of IL-10 without the suppression of Th2 immune responses is not sufficient to suppress reactivity.

In summary, administering allergen with NE induces long-term immune changes that provide protection from allergen challenge for months after discontinuation of therapy. The cytokine profiles in allergic mice after immunization with the NE vaccines showed Th2 cytokines remained significantly suppressed and Th1/Th17 allergenspecific responses were augmented. This suggests allergen/NE immunization reprograms the immune system toward a balanced immune response that may have more lasting effects in suppressing allergic disease than traditional allergen desensitization immunotherapy.

\section{ACKNOWLEDGMENTS}

This project has been funded by a Food Allergy Research and Education New Investigator Award, Michigan Food Allergy Research Accelerator (M-FARA), and a generous gift from Robert and Caren Vondell.

\section{CONFLICTS OF INTEREST}

JRB holds stock in Blue Willow Biologics and is an inventor of the nanoemulsion technology that the University has licensed to Blue Willow Biologics. One of these technologies is involved in this research. JRB and JJO are inventors on a patent application that has been submitted based on this technology (PCT/US2015/054943). The other authors declare no conflicts of interest.

\section{AUTHOR CONTRIBUTIONS}

JJO and JRB designed the study. JJO, JJL, KWL, HKL, AMM, and TDT performed experiments and interpreted data. JJO and JRB wrote the manuscript.

\section{ORCID}

Jessica J. O'Konek (iD https://orcid.org/0000-0002-1455-0586

James R. Baker (iD https://orcid.org/0000-0002-4478-1932 


\section{REFERENCES}

1. Sicherer SH, Sampson HA. Food allergy: epidemiology, pathogenesis, diagnosis, and treatment. J Allergy Clin Immunol. 2014;133(2):291-307.

2. Gupta RS, Springston EE, Warrier MR, et al. The prevalence, severity, and distribution of childhood food allergy in the United States. Pediatrics. 2011;128(1). https://doi.org/10.1542/peds.2011-0204

3. Panel NI-SE, Boyce JA, Assa'ad A, et al. Guidelines for the diagnosis and management of food allergy in the United States: report of the NIAID-sponsored expert panel. J Allergy Clin Immunol. 2010;126(6 Suppl):S1-S58.

4. Saarinen KM, Pelkonen AS, Makela MJ, Savilahti E. Clinical course and prognosis of cow's milk allergy are dependent on milk-specific IgE status. J Allergy Clin Immunol. 2005;116(4):869-875.

5. Host A, Halken S, Jacobsen HP, Christensen AE, Herskind AM, Plesner K. Clinical course of cow's milk protein allergy/intolerance and atopic diseases in childhood. Pediatr Allergy Immunol. 2002;13(Suppl 15):23-28.

6. Feuille E, Nowak-Wegrzyn A. Allergen-specific immunotherapies for food allergy. Allergy Asthma Immunol Res. 2018;10(3):189-206.

7. Lanser BJ, Leung D. The current state of epicutaneous immunotherapy for food allergy: a comprehensive review. Clin Rev Allergy Immunol. 2018;55(2):153-161.

8. Wood RA. Food allergen immunotherapy: current status and prospects for the future. J Allergy Clin Immunol. 2016;137(4):973-982.

9. Bird JA, Spergel JM, Jones SM, et al. Efficacy and safety of AR101 in oral immunotherapy for peanut allergy: results of ARC001, a randomized, double-blind, placebo-controlled phase 2 clinical trial. Allergy Clin Immunol Pract. 2018;6(2):476-485.

10. Investigators PGoC, Vickery BP, Vereda A, et al. AR101 oral immunotherapy for peanut allergy. N Engl J Med. 2018;379(21):1991-2001.

11. Taniuchi S, Takahashi M, Soejima K, Hatano Y, Minami H. Immunotherapy for cow's milk allergy. Hum Vaccin Immunother 2017;13(10):2443-2451.

12. Wood RA, Kim JS, Lindblad R, et al. A randomized, double-blind, placebo-controlled study of omalizumab combined with oral immunotherapy for the treatment of cow's milk allergy. J Allergy Clin Immunol. 2016;137(4):1103-1110.

13. Takahashi M, Taniuchi S, Soejima K, Hatano Y, Yamanouchi S, Kaneko K. Two-weeks-sustained unresponsiveness by oral immunotherapy using microwave heated cow's milk for children with cow's milk allergy. Allergy Asthma Clin Immunol. 2016;12(1):44.

14. Yanagida N, Sato S, Asaumi T, Okada Y, Ogura K, Ebisawa M. A singlecenter, case-control study of low-dose-induction oral immunotherapy with cow's milk. Int Arch Allergy Immunol. 2015;168(2):131-137.

15. Galli SJ, Tsai M. IgE and mast cells in allergic disease. Nat Med. 2012;18(5):693-704.

16. Kim HY, DeKruyff RH, Umetsu DT. The many paths to asthma: phenotype shaped by innate and adaptive immunity. Nat Immunol. 2010;11(7):577-584.

17. Akdis M, Akdis CA. Mechanisms of allergen-specific immunotherapy: multiple suppressor factors at work in immune tolerance to allergens. J Allergy Clin Immunol. 2014;133(3):621-631.

18. Bielinska $\mathrm{AU}$, O'Konek JJ, Janczak KW, Baker JR Jr. Immunomodulation of TH2 biased immunity with mucosal administration of nanoemulsion adjuvant. Vaccine. 2016;34(34):4017-4024.

19. O'Konek JJ, Landers JJ, Janczak KW, et al. Nanoemulsion adjuvant-driven redirection of $\mathrm{TH} 2$ immunity inhibits allergic reactions in murine models of peanut allergy. J Allergy Clin Immunol. 2018;141(6):2121-2131.

20. Makidon PE, Bielinska AU, Nigavekar SS, et al. Pre-clinical evaluation of a novel nanoemulsion-based hepatitis $B$ mucosal vaccine. PLoS One 2008;3(8):e2954.
21. Myc A, Kukowska-Latallo JF, Bielinska AU, et al. Development of immune response that protects mice from viral pneumonitis after a single intranasal immunization with influenza $A$ virus and nanoemulsion. Vaccine. 2003;21(25-26):3801-3814.

22. Ahrens R, Osterfeld H, Wu D, et al. Intestinal mast cell levels control severity of oral antigen-induced anaphylaxis in mice. Am J Pathol. 2012;180(4):1535-1546.

23. Li XM, Serebrisky D, Lee SY, et al. A murine model of peanut anaphylaxis: $\mathrm{T}$ - and $\mathrm{B}$-cell responses to a major peanut allergen mimic human responses. J Allergy Clin Immunol. 2000;106(1 Pt 1):150-158.

24. Rodriguez B, Prioult G, Hacini-Rachinel F, et al. Infant gut microbiota is protective against cow's milk allergy in mice despite immature ileal T-cell response. FEMS Microbiol Ecol. 2012;79(1):192-202.

25. Classen DC, Morningstar JM, Shanley JD. Detection of antibody to murine cytomegalovirus by enzyme-linked immunosorbent and indirect immunofluorescence assays. J Clin Microbiol. 1987;25(4):600-604.

26. Frey A, Di Canzio J, Zurakowski D. A statistically defined endpoint titer determination method for immunoassays. J Immunol Methods. 1998;221(1-2):35-41.

27. Friend DS, Ghildyal N, Austen KF, Gurish MF, Matsumoto R, Stevens RL. Mast cells that reside at different locations in the jejunum of mice infected with Trichinella spiralis exhibit sequential changes in their granule ultrastructure and chymase phenotype. J Cell Biol. 1996;135(1):279-290.

28. Brandt EB, Strait RT, Hershko D, et al. Mast cells are required for experimental oral allergen-induced diarrhea. J Clin Invest. 2003;112(11):1666-1677.

29. Chen CY, Lee JB, Liu B, et al. Induction of interleukin-9-producing mucosal mast cells promotes susceptibility to IgE-mediated experimental food allergy. Immunity. 2015;43(4):788-802.

30. Sindher SB, Long A, Acharya S, Sampath V, Nadeau KC. The use of biomarkers to predict aero-allergen and food immunotherapy responses. Clin Rev Allergy Immunol. 2018;55(2):190-204.

31. Gupta RS, Walkner MM, Greenhawt M, et al. Food allergy sensitization and presentation in siblings of food allergic children. J Allergy Clin Immunol Pract. 2016;4(5):956-962.

32. Flicker S, Valenta R. Renaissance of the blocking antibody concept in type I allergy. Int Arch Allergy Immunol. 2003;132(1):13-24.

33. Strait RT, Morris SC, Finkelman FD. IgG-blocking antibodies inhibit IgE-mediated anaphylaxis in vivo through both antigen interception and Fc gamma RIlb cross-linking. J Clin Invest. 2006;116(3):833-841.

34. Daeron M, Malbec O, Latour S, Arock M, Fridman WH. Regulation of high-affinity IgE receptor-mediated mast cell activation by murine low-affinity IgG receptors. J Clin Invest. 1995;95(2):577-585.

35. Ravetch JV, Bolland S. IgG Fc receptors. Annu Rev Immunol. 2001;19:275-290.

36. Mondoulet L, Dioszeghy V, Vanoirbeek JA, Nemery B, Dupont C, Benhamou PH. Epicutaneous immunotherapy using a new epicutaneous delivery system in mice sensitized to peanuts. Int Arch Allergy Immunol. 2011;154(4):299-309.

37. Mondoulet L, Dioszeghy V, Ligouis M, Dhelft V, Dupont C, Benhamou PH. Epicutaneous immunotherapy on intact skin using a new delivery system in a murine model of allergy. Clin Exp Allergy. 2010;40(4):659-667.

38. Tordesillas L, Mondoulet L, Blazquez AB, Benhamou PH, Sampson HA, Berin MC. Epicutaneous immunotherapy induces gastrointestinal $\mathrm{LAP}(+)$ regulatory $T$ cells and prevents food-induced anaphylaxis. J Allergy Clin Immunol. 2017;139(1):189-201.

39. Vonk MM, Wagenaar L, Pieters R, et al. The efficacy of oral and subcutaneous antigen-specific immunotherapy in murine cow's milk- and peanut allergy models. Clin Transl Allergy. 2017;7:35.

40. Kulis M, Macqueen I, Li Y, Guo R, Zhong XP, Burks AW. Pepsinized cashew proteins are hypoallergenic and immunogenic and provide 
effective immunotherapy in mice with cashew allergy. J Allergy Clin Immunol. 2012;130(3):716-723.

41. Yang M, Yang C, Mine Y. Multiple T cell epitope peptides suppress allergic responses in an egg allergy mouse model by the elicitation of forkhead box transcription factor 3- and transforming growth factor-beta-associated mechanisms. Clin Exp Allergy. 2010;40(4):668-678.

42. Rupa P, Mine Y. Oral immunotherapy with immunodominant T-cell epitope peptides alleviates allergic reactions in a Balb/c mouse model of egg allergy. Allergy. 2012;67(1):74-82.

43. Wai CY, Leung NY, Leung PS, Chu KH. T cell epitope immunotherapy ameliorates allergic responses in a murine model of shrimp allergy. Clin Exp Allergy. 2016;46(3):491-503.

44. Smaldini PL, Trejo F, Cohen JL, Piaggio E, Docena GH. Systemic IL-2/anti-IL-2Ab complex combined with sublingual immunotherapy suppresses experimental food allergy in mice through induction of mucosal regulatory T cells. Allergy. 2018;73(4):885-895.

45. Zhu FG, Kandimalla ER, Yu D, Agrawal S. Oral administration of a synthetic agonist of Toll-like receptor 9 potently modulates peanutinduced allergy in mice. J Allergy Clin Immunol. 2007;120(3):631-637.

46. Leonard SA, Martos G, Wang W, Nowak-Wegrzyn A, Berin MC. Oral immunotherapy induces local protective mechanisms in the gastrointestinal mucosa. J Allergy Clin Immunol. 2012;129(6):1579-1587.

47. Vickery BP, Scurlock AM, Kulis M, et al. Sustained unresponsiveness to peanut in subjects who have completed peanut oral immunotherapy. J Allergy Clin Immunol. 2014;133(2):468-475.

48. Burks AW, Jones SM, Wood RA, et al. Oral immunotherapy for treatment of egg allergy in children. N Engl J Med. 2012;367(3):233-243.

49. Akdis CA, Akdis M. Advances in allergen immunotherapy: aiming for complete tolerance to allergens. Sci Transl Med. 2015;7(280):280ps 6-280ps6.
50. Palomares O, Akdis M, Martin-Fontecha M, Akdis CA. Mechanisms of immune regulation in allergic diseases: the role of regulatory $T$ and B cells. Immunol Rev. 2017;278(1):219-236.

51. Schulten V, Tripple V, Sidney J, et al. Association between specific timothy grass antigens and changes in TH1- and TH2-cell responses following specific immunotherapy. J Allergy Clin Immunol. 2014;134(5):1076-1083.

52. Schulten V, Tripple V, Aasbjerg K, et al. Distinct modulation of allergic $T$ cell responses by subcutaneous vs. sublingual allergen-specific immunotherapy. Clin Exp Allergy. 2016;46(3):439-448.

\section{SUPPORTING INFORMATION}

Additional supporting information may be found online in the Supporting Information section at the end of the article.

How to cite this article: O'Konek JJ, Landers JJ, Janczak KW, et al. Intranasal nanoemulsion vaccine confers long-lasting immunomodulation and sustained unresponsiveness in a murine model of milk allergy. Allergy. 2020;75:872-881. https ://doi.org/10.1111/all.14064 